\title{
Scaled particle theory for a hard spherocylinder fluid in a disordered porous medium: Carnahan-Starling and Parsons-Lee corrections
}

\author{
M.F. Holovko, V.I. Shmotolokha \\ Institute for Condensed Matter Physics of the National Academy of Sciences of Ukraine, \\ 1 Svientsitskii St., 79011 Lviv, Ukraine
}

Received February 1, 2018

\begin{abstract}
The scaled particle theory (SPT) approximation is applied for the study of the influence of a porous medium on the isotropic-nematic transition in a hard spherocylinder fluid. Two new approaches are developed in order to improve the description in the case of small lengths of spherocylinders. In one of them, the so-called SPT-CS-PL approach, the Carnahan-Starling (CS) correction is introduced to improve the description of thermodynamic properties of the fluid, while the Parsons-Lee (PL) correction is introduced to improve the orientational ordering. The second approach, the so-called SPT-PL approach, is connected with generalization of the PL theory to anisotropic fluids in disordered porous media. The phase diagram is obtained from the bifurcation analysis of a nonlinear integral equation for the singlet distribution function and from the thermodynamic equilibrium conditions. The results obtained are compared with computer simulation data. Both ways and both approaches considerably improve the description in the case of spherocylinder fluids with smaller spherocylinder lengths. We did not find any significant differences between the results of the two developed approaches. We found that the bifurcation analysis slightly overestimates and the thermodynamical analysis underestimates the predictions of the computer simulation data. A porous medium shifts the phase diagram to smaller densities of the fluid and does not change the type of the transition.
\end{abstract}

Key words: hard spherocylinder fluid, porous material, scaled particle theory, isotropic-nematic transition, Parsons-Lee theory, Carnahan-Starling correction

PACS: $61.20 . G y, 61.43 . G y$

\section{Introduction}

A hard spherocylinder fluid is one of the simplest popular models widely used for the description of isotropic-nematic phase transitions in the theory of liquid crystals [1]. During this transition the fluid separates into two phases with two different densities. The phase with the lower density is the isotropic one and in this phase the distribution of molecular orientations is uniform. The other phase with the higher density is the nematic one and in this phase molecular orientations are strongly ordered. This phase separation was first explained by Onsager [2] nearly seventy years ago as a result of competition between the orientational entropy that favours disorder and the entropy effect associated with the orientationaldependent excluded volume of spherocylinder-like particles that favours order. Onsager's treatment of the isotropic-nematic transition was given for a very specific model of a hard spherocylinder fluid in which the length of spherocylinder $L_{1} \rightarrow \infty$ and the diameter $D_{1} \rightarrow 0$ in such a way that the non-dimensional density of the fluid $c_{1}=\frac{1}{4} \pi \rho_{1} L_{1}^{2} D_{1}$ is fixed, where $\rho_{1}=\frac{N_{1}}{V}, N_{1}$ is the number of spherocylinders, $V$ is the volume of the system. The Onsager theory is based on the low-density expansion of the free energy functional truncated at the second virial coefficient level. The result obtained for such a model in this description is exact [1].

The application of the scaled particle theory (SPT) previously developed for a hard-sphere fluid [3, 4] 
provides an efficient approximate way to incorporate the higher order contributions neglected in the Onsager theory. As a result, it was possible to generalize the Onsager theory for the description of a more realistic model of the hard spherocylinder fluid with a finite value of the length of spherocylinder $L_{1}$ and a nonzero value of the diameter $D_{1}[5,-7]$. We note that for thermodynamic properties of a hard sphere fluid, the SPT produces the same result as the Percus-Yevick theory [8, 9]. A basic defect of such a description is known to appear at higher densities where the theory needs some improvement, such as a semi-empirical Carnahan-Starling (CS) correction [10, 11]. Recently the SPT was applied for the description of isotropic-nematic phase transition in a mixture of hard spheres and hard spherocylinders. By comparison with the corresponding computer simulation data [12, 14] it was shown that the accuracy of the SPT description reduces with a decreasing length $L_{1}$ of spherocylinder. Such a bad accuracy of SPT description can be improved by CS correction. An alternative to SPT way of improvement of the Onsager theory is the Parsons-Lee (PL) approach [15.17] which is based on the mapping of the properties of the hard spherocylinder fluid to those of the hard sphere fluid. The application of the CS theory to the hard sphere system in the PL theory leads to a correct description of the isotropic-nematic transition in the hard spherocylinder fluid even at small lengths $L_{1}$ of spherocylinders [16].

During the last decade the scaled particle theory was extended to generalize for the description of a hard sphere fluid in disordered porous media [18-24]. The obtained results were generalized for the fluid of hard convex body particles in disordered porous media [25] and was used for the study of the influence of porous media on the isotropic-nematic transition in a hard spherocylinder fluid in disordered porous media [26, 27]. It was shown that a porous medium shifts the isotropic-nematic phase transition to smaller fluid densities. However, similar to the bulk case, the accuracy of the developed SPT description reduces with a decreasing spherocylinder length. In this paper, in order to improve the SPT description of a hard spherocylinder fluid in disordered porous media we will introduce two types of corrections. The first one is the CS correction which improves the description at higher densities of the fluid. The second one corrects the description of the orientational ordering in a hard spherocylinder fluid at higher densities. This correction is formulated by comparison of the constants in the integral equation for the singlet distribution function of hard spherocylinders in the SPT approach and in the PL theory. In this paper, the CS and PL corrections constitute the improvement of the SPT description of a hard spherocylinder fluid in disordered porous media. In parallel to the SPT approach, we also consider the generalization of the PL theory for a hard spherocylinder fluid in disordered porous media. It is shown that both approaches provide a correct description of the isotropic-nematic phase transition in a hard spherocylinder fluid in disordered porous media including the hard spherocylinder fluids with small lengths of spherocylinders.

The paper is arranged as follows. In section 2 we give a brief review of the application of the SPT approach for a hard spherocylinder fluid in disordered porous media. An improvement of the SPT description with the CS and the PL corrections is presented in section 3. In section 4 generalization of the PL theory for a hard spherocylinder fluid in disordered porous media is presented. The results and discussion are presented in section 5 . We conclude in section 6

\section{SPT for hard spherocylinder fluids in disordered porous media}

In this section we present a short review of the SPT for hard spherocylinder fluids in disordered porous media. The basic idea of the SPT lies in the insertion of an additional hard spherocylinder with the scaling diameter $D_{\mathrm{s}}$ and the scaling length $L_{\mathrm{s}}$ into a fluid in such a way that

$$
D_{\mathrm{s}}=\lambda_{\mathrm{s}} D_{1}, \quad L_{\mathrm{s}}=\alpha_{\mathrm{s}} L_{1},
$$

where $D_{1}$ and $L_{1}$ are the diameter and the length of fluid spherocylinder, respectively. In the presence of porous media, the excess of chemical potential for the small scaled particle in a spherocylinder fluid confined in a matrix can be written in the form [26]

$$
\beta \mu_{\mathrm{s}}^{\mathrm{ex}}=-\ln p_{0}\left(\alpha_{\mathrm{s}}, \lambda_{\mathrm{s}}\right)-\ln \left\{1-\frac{\eta_{1}}{V_{1} p_{0}\left(\alpha_{\mathrm{s}}, \lambda_{\mathrm{s}}\right)}\left[\frac{\pi}{6} D_{1}^{3}\left(1+\lambda_{\mathrm{s}}\right)^{3}+\frac{\pi}{4} D_{1}^{2} L_{1}\left(1+\lambda_{\mathrm{s}}\right)^{2}\left(1+\alpha_{\mathrm{s}}\right)\right.\right.
$$




$$
\left.\left.+\frac{\pi}{4} D_{1} L_{1}^{2}\left(1+\lambda_{\mathrm{s}}\right) \alpha_{\mathrm{s}} \iint f\left(\Omega_{1}\right) f\left(\Omega_{2}\right) \sin \gamma\left(\Omega_{1}, \Omega_{2}\right) \mathrm{d} \Omega_{1} \mathrm{~d} \Omega_{2}\right]\right\}
$$

where $\beta=\frac{1}{k T}, k$ is the Boltzmann constant, $T$ is temperature, $\eta_{1}=\rho_{1} V_{1}$ is the fluid packing fraction, $\rho_{1}$ is the fluid density, $V_{1}$ is the volume of spherocylinder; $p_{0}\left(\alpha_{\mathrm{s}}, \lambda_{\mathrm{s}}\right)$ is the probability to find a cavity created by a scale particle in the empty matrix and is defined by the excess of a chemical potential $\mu_{\mathrm{s}}^{0}$ of the scale particle in the limit of the infinite dilution of a fluid; $\Omega=(\vartheta, \varphi)$ is the orientation of particles defined by the angles $\vartheta$ and $\varphi ; \mathrm{d} \Omega=\frac{1}{4 \pi} \sin \vartheta \mathrm{d} \vartheta \mathrm{d} \varphi$ is the normalized angle element; $\gamma\left(\Omega_{1}, \Omega_{2}\right)$ is an angle between orientational vectors of two molecules; $f(\Omega)$ is the singlet orientational distribution function normalized in such a way that

$$
\int f(\Omega) \mathrm{d} \Omega=1 .
$$

For a large scale particle, the excess of chemical potential is given by a thermodynamic expression that can be presented in the form:

$$
\beta \mu_{\mathrm{s}}^{\mathrm{ex}}=w\left(\alpha_{\mathrm{s}}, \lambda_{\mathrm{s}}\right)+\beta P V_{\mathrm{s}} / p_{0}\left(\lambda_{\mathrm{s}}, \alpha_{\mathrm{s}}\right),
$$

where $P$ is the pressure of the fluid, $V_{\mathrm{s}}$ is the volume of the scaled particle, the multiplier $1 / p_{0}\left(\lambda_{\mathrm{s}}, \alpha_{\mathrm{s}}\right)$ appears due to an excluded volume confined by matrix particles and can be considered as a probability to find a cavity created by a scaled particle in the absence of fluid particles. The probability $p_{0}\left(\lambda_{\mathrm{s}}, \alpha_{\mathrm{s}}\right)$ is directly related to two different types of porosity introduced by us in [10, 12, 14, 26].

The first one corresponds to the geometrical porosity

$$
\phi_{0}=p_{0}\left(\alpha_{\mathrm{s}}=\lambda_{\mathrm{s}}=0\right),
$$

characterizing the free volume for a fluid. The second type of porosity corresponds to the case $\lambda_{\mathrm{s}}=\alpha_{\mathrm{s}}=1$ and leads to the thermodynamic porosity

$$
\phi=p_{0}\left(\alpha_{\mathrm{s}}=\lambda_{\mathrm{s}}=1\right)=\exp \left(-\beta \mu_{1}^{0}\right),
$$

defined by the excess chemical potential of fluid particles $\mu_{1}^{0}$ in the limit of infinite dilution. It characterizes the adsorption of a fluid in the empty matrix. According to the ansatz of the SPT [5--7, 26] $w\left(\lambda_{\mathrm{s}}, \alpha_{\mathrm{s}}\right)$ can be presented in the form:

$$
w\left(\lambda_{\mathrm{s}}, \alpha_{\mathrm{s}}\right)=w_{00}+w_{10} \lambda_{\mathrm{s}}+w_{01} \alpha_{\mathrm{s}}+w_{11} \alpha_{\mathrm{s}} \lambda_{\mathrm{s}}+\frac{w_{20} \lambda_{\mathrm{s}}^{2}}{2},
$$

where the coefficients of this expansion can be found from the continuity of the excess chemical potential given in 2.2 and 2.4, as well as from the corresponding derivatives $\partial \mu_{\mathrm{s}}^{\mathrm{ex}} / \partial \lambda_{\mathrm{s}}, \partial \mu_{\mathrm{s}}^{\mathrm{ex}} / \partial \alpha_{\mathrm{s}}, \partial^{2} \mu_{\mathrm{s}}^{\mathrm{ex}} / \partial \alpha_{\mathrm{s}} \partial \lambda_{\mathrm{s}}$ and $\partial^{2} \mu_{\mathrm{s}}^{\mathrm{ex}} / \partial \lambda_{\mathrm{s}}^{2}$. As a result, one derives the coefficients as follows:

$$
\begin{gathered}
w_{00}=-\ln \left(1-\eta_{1} / \phi_{0}\right), \\
w_{10}=\frac{\eta_{1} / \phi_{0}}{1-\eta_{1} / \phi_{0}}\left(\frac{6 \gamma_{1}}{3 \gamma_{1}-1}-\frac{p_{0 \lambda}^{\prime}}{\phi_{0}}\right), \\
w_{01}=\frac{\eta_{1} / \phi_{0}}{1-\eta_{1} / \phi_{0}}\left[\frac{3\left(\gamma_{1}-1\right)}{3 \gamma_{1}-1}+\frac{3\left(\gamma_{1}-1\right)^{2}}{3 \gamma_{1}-1} \tau(f)-\frac{p_{0 \alpha}^{\prime}}{\phi_{0}}\right], \\
w_{11}=\frac{\eta_{1} / \phi_{0}}{1-\eta_{1} / \phi_{0}}\left[\frac{6\left(\gamma_{1}-1\right)}{3 \gamma_{1}-1}+\frac{3\left(\gamma_{1}-1\right)^{2} \tau(f)}{3 \gamma_{1}-1}-\frac{p_{0 \alpha \lambda}^{\prime \prime}}{\phi_{0}}\right. \\
\left.+2 \frac{p_{0 \alpha}^{\prime} p_{0 \lambda}^{\prime}}{\phi_{0}^{2}}-\frac{3\left(\gamma_{1}-1\right)+3\left(\gamma_{1}-1\right)^{2} \tau(f)}{3 \gamma_{1}-1} \frac{p_{0 \lambda}^{\prime}}{\phi_{0}}-\frac{6 \gamma_{1}}{3 \gamma_{1}-1} \frac{p_{0 \alpha}^{\prime}}{\phi_{0}}\right] \\
+\left(\frac{\eta_{1} / \phi_{0}}{1-\eta_{1} / \phi_{0}}\right)^{2}\left(\frac{6 \gamma_{1}}{3 \gamma_{1}-1}-\frac{p_{0 \lambda}^{\prime}}{\phi_{0}}\right)\left[\frac{3\left(\gamma_{1}-1\right)}{3 \gamma_{1}-1}+\frac{3\left(\gamma_{1}-1\right)^{2} \tau(f)}{3 \gamma_{1}-1}-\frac{p_{0 \alpha}^{\prime}}{\phi_{0}}\right],
\end{gathered}
$$




$$
\begin{aligned}
w_{20} & =\frac{\eta_{1} / \phi_{0}}{1-\eta_{1} / \phi_{0}}\left[\frac{6\left(1+\gamma_{1}\right)}{3 \gamma_{1}-1}-\frac{12 \gamma_{1}}{3 \gamma_{1}-1} \frac{p_{0 \lambda}^{\prime}}{\phi_{0}}+2\left(\frac{p_{0 \lambda}^{\prime}}{\phi_{0}}\right)^{2}-\frac{p_{0 \lambda \lambda}^{\prime \prime}}{\phi_{0}}\right] \\
& +\left(\frac{\eta_{1} / \phi_{0}}{1-\eta_{1} / \phi_{0}}\right)^{2}\left(\frac{6 \gamma_{1}}{3 \gamma_{1}-1}-\frac{p_{0 \lambda}^{\prime}}{\phi_{0}}\right)^{2},
\end{aligned}
$$

where

$$
\begin{gathered}
\gamma_{1}=1+\frac{L_{1}}{D_{1}}, \\
\tau(f)=\frac{4}{\pi} \iint f\left(\Omega_{1}\right) f\left(\Omega_{2}\right) \sin \gamma\left(\Omega_{1}, \Omega_{2}\right) \mathrm{d} \Omega_{1} \mathrm{~d} \Omega_{2} .
\end{gathered}
$$

Setting $\alpha_{\mathrm{s}}=\lambda_{\mathrm{s}}=1$ in the equation (2.4) leads to the expression

$$
\beta\left(\mu_{1}^{\mathrm{ex}}-\mu_{1}^{0}\right)=-\ln \left(1-\eta_{1} / \phi_{0}\right)+A(\tau(f)) \frac{\eta_{1} / \phi_{0}}{1-\eta_{1} / \phi_{0}}+B(\tau(f)) \frac{\left(\eta_{1} / \phi_{0}\right)^{2}}{\left(1-\eta_{1} / \phi_{0}\right)^{2}},
$$

where the coefficients $A(\tau(f))$ and $B(\tau(f))$ define the porous medium structure and the expressions for them are as follows:

$$
\begin{array}{r}
A(\tau(f))=6+\frac{6\left(\gamma_{1}-1\right)^{2} \tau(f)}{3 \gamma_{1}-1}-\frac{p_{0 \lambda}^{\prime}}{\phi_{0}}\left[4+\frac{3\left(\gamma_{1}-1\right)^{2} \tau(f)}{3 \gamma_{1}-1}\right] \\
-\frac{p_{0 \alpha}^{\prime}}{\phi_{0}}\left(1+\frac{6 \gamma_{1}}{3 \gamma_{1}-1}\right)-\frac{p_{0 \alpha \lambda}^{\prime \prime}}{\phi_{0}}-\frac{1}{2} \frac{p_{0 \lambda \lambda}^{\prime \prime}}{\phi_{0}}+2 \frac{p_{0 \alpha}^{\prime} p_{0 \lambda}^{\prime}}{\phi_{0}^{2}}+\left(\frac{p_{0 \lambda}^{\prime}}{\phi_{0}}\right)^{2} \\
B(\tau(f))=\left(\frac{6 \gamma_{1}}{3 \gamma_{1}-1}-\frac{p_{0 \lambda}^{\prime}}{\phi_{0}}\right)\left[\frac{3\left(2 \gamma_{1}-1\right)}{3 \gamma_{1}-1}+\frac{3\left(\gamma_{1}-1\right)^{2} \tau(f)}{3 \gamma_{1}-1}-\frac{p_{0 \alpha}^{\prime}}{\phi_{0}}-\frac{1}{2} \frac{p_{0 \lambda}^{\prime}}{\phi_{0}}\right]
\end{array}
$$

where $p_{0 \lambda}^{\prime}=\frac{\partial p_{0}\left(\alpha_{\mathrm{s}}, \lambda_{\mathrm{s}}\right)}{\partial \lambda_{\mathrm{s}}}, p_{0 \alpha}^{\prime}=\frac{\partial p_{0}\left(\alpha_{\mathrm{s}}, \lambda_{\mathrm{s}}\right)}{\partial \alpha_{\mathrm{s}}}, p_{0 \alpha \lambda}^{\prime \prime}=\frac{\partial^{2} p_{0}\left(\alpha_{\mathrm{s}}, \lambda_{\mathrm{s}}\right)}{\partial \alpha_{\mathrm{s}} \partial \lambda_{\mathrm{s}}}, p_{0 \lambda \lambda}^{\prime \prime}=\frac{\partial^{2} p_{0}\left(\alpha_{\mathrm{s}}, \lambda_{\mathrm{s}}\right)}{\partial \lambda_{\mathrm{s}}^{2}}$ are the corresponding derivatives at $\alpha=\lambda=0$. Using the Gibbs-Duhem equation $\left(\frac{\partial P}{\partial \rho_{1}}\right)_{T}=\rho_{1}\left(\frac{\partial \mu_{1}}{\partial \rho_{1}}\right)_{T}$, which relates the pressure $P$ of a fluid to its total chemical potential $\mu_{1}=\ln \left(\eta_{1}\right)+\mu_{1}^{0}+\mu_{1}^{\text {ex }}$ one derives the fluid compressibility in the form

$$
\begin{aligned}
& \beta\left(\frac{\partial P}{\partial \rho_{1}}\right)_{T}=\frac{1}{\left(1-\eta_{1} / \phi\right)}+[1+A(\tau(f))] \frac{\eta_{1} / \phi_{0}}{\left(1-\eta_{1} / \phi\right)\left(1-\eta_{1} / \phi_{0}\right)} \\
& +[A(\tau(f))+2 B(\tau(f))] \frac{\left(\eta_{1} / \phi_{0}\right)^{2}}{\left(1-\eta_{1} / \phi\right)\left(1-\eta_{1} / \phi_{0}\right)^{2}}+2 B(\tau(f)) \frac{\left(\eta_{1} / \phi_{0}\right)^{3}}{\left(1-\eta_{1} / \phi\right)\left(1-\eta_{1} / \phi_{0}\right)^{3}} .
\end{aligned}
$$

From expression (2.18) it is possible to obtain the chemical expression and the pressure of the fluid in SPT2 approach [10, 12, 26]. The expression (2.18) at higher densities has two divergences, which appear at $\eta_{1}=\phi$ and $\eta_{1}=\phi_{0}$, respectively. Since $\phi<\phi_{0}$, the divergence at $\eta_{1}=\phi$ occurs at lower densities than the second one and, therefore, should be removed. Different corrections and improvements of SPT2 approach were proposed in [10, $12,14,26]$. The first corrections were considered in [10] where based on SPT2, four different approximations were proposed. The best one is the SPT2b approximation which was derived replacing $\phi$ by $\phi_{0}$ everywhere in (2.18) except the first term. However, this term has a divergence at $\eta_{1}=\phi$ and due to this, some other approximations were proposed in [11, 12, 14, 26]. One of them called SPT2b1 can be obtained from the expression for the chemical potential in SPT2b approach by removing the divergence at $\eta_{1}=\phi$ through the expansion of the logarithmic term in the SPT2b expression for the chemical potential as follows:

$$
-\ln \left(1-\eta_{1} / \phi\right) \approx-\ln \left(1-\eta_{1} / \phi\right)+\frac{\eta_{1}\left(\phi_{0}-\phi\right)}{\phi_{0} \phi\left(1-\eta_{1} / \phi_{0}\right)} .
$$


Therefore, one obtains the expressions for the chemical potential and pressure within the SPT2b1 approximation as follows:

$$
\begin{gathered}
{\left[\beta\left(\mu_{1}^{\mathrm{ex}}-\mu_{1}^{0}\right)\right]^{\mathrm{SPT} 2 \mathrm{~b} 1}=\sigma(f)-\ln \left(1-\eta_{1} / \phi_{0}\right)+[1+A(\tau(f))] \frac{\eta_{1} / \phi_{0}}{1-\eta_{1} / \phi_{0}}+\frac{\eta_{1}\left(\phi_{0}-\phi\right)}{\phi_{0} \phi\left(1-\eta_{1} / \phi_{0}\right)}} \\
+\frac{1}{2}[A(\tau(f))+2 B(\tau(f))] \frac{\left(\eta_{1} / \phi_{0}\right)^{2}}{\left(1-\eta_{1} / \phi_{0}\right)^{2}}+\frac{2}{3} B(\tau(f)) \frac{\left(\eta_{1} / \phi_{0}\right)^{3}}{\left(1-\eta_{1} / \phi_{0}\right)^{3}} \\
\left(\frac{\beta P}{\rho_{1}}\right)^{\mathrm{SPT} 2 \mathrm{~b} 1}=\frac{1}{1-\eta_{1} / \phi_{0}} \frac{\phi_{0}}{\phi}+\left(\frac{\phi_{0}}{\phi}-1\right) \frac{\phi_{0}}{\eta_{1}} \ln \left(1-\eta_{1} / \phi_{0}\right) \\
+\frac{A(\tau(f))}{2} \frac{\eta_{1} / \phi_{0}}{\left(1-\eta_{1} / \phi_{0}\right)^{2}}+\frac{2 B(\tau(f))}{3} \frac{\left(\eta_{1} / \phi_{0}\right)^{2}}{\left(1-\eta_{1} / \phi_{0}\right)^{3}}
\end{gathered}
$$

where

$$
\sigma(f)=\int f(\Omega) \ln f(\Omega) \mathrm{d} \Omega
$$

is the entropic term.

Some other approximations which include the third type of porosity $\phi^{*}$ defined by the maximum value of packing fraction of a fluid in a porous media are analyzed in [11, 12, 14]. However, in this paper we restrict our consideration to the SPT2b1 approximation which is quite accurate at small, intermediate and higher fluid densities.

From the thermodynamic relationship

$$
\frac{\beta F}{V}=\beta \mu_{1} \rho_{1}-\beta P,
$$

one can obtain an expression for the free energy. Within the SPT2b1 approximation, the free energy of a confined fluid is as follows:

$$
\begin{aligned}
& \left(\frac{\beta F}{N}\right)^{\mathrm{SPT2b1}}=\sigma(f)+\ln \frac{\eta_{1}}{\phi}-1-\ln \left(1-\eta_{1} / \phi_{0}\right)+\left(1-\frac{\phi_{0}}{\phi}\right)\left[1+\frac{\phi_{0}}{\eta_{1}} \ln \left(1-\eta_{1} / \phi_{0}\right)\right] \\
& +\frac{A(\tau(f))}{2} \frac{\eta_{1} / \phi_{0}}{1-\eta_{1} / \phi_{0}}+\frac{B(\tau(f))}{3}\left(\frac{\eta_{1} / \phi_{0}}{1-\eta_{1} / \phi_{0}}\right)^{2} .
\end{aligned}
$$

The singlet orientational distribution function $f(\Omega)$ can be obtained from the minimization of the free energy with respect to variations of this distribution. This procedure leads to the nonlinear integral equation

$$
\ln f\left(\Omega_{1}\right)+\lambda+\frac{8}{\pi} C \int f\left(\Omega^{\prime}\right) \sin \gamma\left(\Omega_{1} \Omega^{\prime}\right) \mathrm{d} \Omega^{\prime}=0
$$

where

$$
C^{\mathrm{SPT} 2 \mathrm{~b} 1}=\frac{\eta_{1} / \phi_{0}}{1-\eta_{1} / \phi_{0}}\left[\frac{3\left(\gamma_{1}-1\right)^{2}}{3 \gamma_{1}-1}\left(1-\frac{p_{0 \lambda}^{\prime}}{2 \phi_{0}}\right)+\frac{\eta_{1} / \phi_{0}}{\left(1-\eta_{1} / \phi_{0}\right)} \frac{\left(\gamma_{1}-1\right)^{2}}{3 \gamma_{1}-1}\left(\frac{6 \gamma_{1}}{3 \gamma_{1}-1}-\frac{p_{0 \lambda}^{\prime}}{\phi_{0}}\right)\right] .
$$

\section{Carnahan-Starling and Parsons-Lee corrections}

As it was already noted at the beginning of this paper, the SPT approach is not accurate enough for higher fluid densities as the length of spherocylinders decreases and the CS correction [11] should be taken into account. The CS correction is generalized for the presence of a porous media. We present the equation of state in the following form:

$$
\left(\frac{\beta P}{\rho_{1}}\right)^{\mathrm{SPT} 2 \mathrm{~b} 1-\mathrm{CS}}=\left(\frac{\beta P}{\rho_{1}}\right)^{\mathrm{SPT} 2 \mathrm{~b} 1}+\left(\frac{\beta \Delta P}{\rho_{1}}\right)^{\mathrm{CS}},
$$


where $\left(\beta P / \rho_{1}\right)^{\mathrm{SPT} 2 \mathrm{~b} 1}$ is given by equation $2.21,\left(\beta \Delta P / \rho_{1}\right)^{\mathrm{CS}}$ is the CS correction which we present in the form

$$
\left(\frac{\beta \Delta P}{\rho_{1}}\right)^{\mathrm{CS}}=-\frac{\left(\eta_{1} / \phi_{0}\right)^{3}}{\left(1-\eta_{1} / \phi_{0}\right)^{3}} .
$$

We present the chemical potentials in a similar form

$$
\left(\beta \mu_{1}\right)^{\mathrm{SPT} 2 \mathrm{~b} 1-\mathrm{CS}}=\left(\beta \mu_{1}\right)^{\mathrm{SPT} 2 \mathrm{~b} 1}+\left(\beta \Delta \mu_{1}\right)^{\mathrm{CS}},
$$

where the correction $\left(\Delta \mu_{1}\right)^{\mathrm{CS}}$ can be obtained from the Gibbs-Duhem equation

$$
\left(\beta \Delta \mu_{1}\right)^{\mathrm{CS}}=\beta \int_{0}^{\eta_{1}} \frac{\mathrm{d} \eta_{1}}{\eta_{1}}\left(\frac{\partial \Delta P}{\partial \rho_{1}}\right)^{\mathrm{CS}} .
$$

As a result,

$$
\left(\beta \Delta \mu_{1}\right)^{\mathrm{CS}}=\ln \left(1-\eta_{1} / \phi_{0}\right)+\frac{\eta_{1} / \phi_{0}}{1-\eta_{1} / \phi_{0}}-\frac{1}{2} \frac{\left(\eta_{1} / \phi_{0}\right)^{2}}{\left(1-\eta_{1} / \phi_{0}\right)^{2}}-\frac{\left(\eta_{1} / \phi_{0}\right)^{3}}{\left(1-\eta_{1} / \phi_{0}\right)^{3}} .
$$

The free energy can also be presented in the form

$$
\left(\frac{\beta F}{N_{1}}\right)^{\mathrm{SPT} 2 \mathrm{~b} 1-\mathrm{CS}}=\left(\frac{\beta F}{N_{1}}\right)^{\mathrm{SPT} 2 \mathrm{~b} 1}+\left(\frac{\beta F}{N_{1}}\right)^{\mathrm{CS}},
$$

where the first term $\left(\beta F / N_{1}\right)^{\mathrm{SPT} 2 \mathrm{~b} 1}$ is given by equation 2.24 and the second term can be found from thermodynamic relation 2.23

$$
\left(\frac{\beta \Delta F}{N_{1}}\right)^{\mathrm{CS}}=\ln \left(1-\eta_{1} / \phi_{0}\right)+\frac{\eta_{1} / \phi_{0}}{1-\eta_{1} / \phi_{0}}-\frac{1}{2} \frac{\left(\eta_{1} / \phi_{0}\right)^{2}}{\left(1-\eta_{1} / \phi_{0}\right)^{2}} .
$$

However, the considered CS correction improves only thermodynamic properties and does not modify the description of orientational ordering which is described by the integral equation $(2.25)$ for the singlet distribution function $f(\Omega)$. In order to improve the description of orientational ordering we should modify the parameter $C$ given by the expression (2.26). This parameter has two terms. The first term appears from the coefficient $A(\tau(f))$ and the second one appears from the coefficient $B(\tau(f))$ in the expression 2.24 for the free energy. By simple comparison of parameter $C$ in the SPT approach and the PL theory for the bulk case we can see that the first term which appears from the coefficient $A(\tau(f))$ in SPT2b1 theory is the same as in the PL approach. Although there are some differences in the second term which appears from the coefficient $B(\tau(f))$, it is possible to have practically the same result for the description of isotropicnematic transition from SPT and PL approaches if we introduce some parameter $\delta$ as a multiplier near the term with $\tau(f)$ in coefficient $B(\tau(f))$. After generalization of this result for the hard spherocylinder fluid in disordered porous media, we can rewrite the expression for $B(\tau(f))$ in the following form

$$
B(\tau(f))=\left(\frac{6 \gamma_{1}}{3 \gamma_{1}-1}-\frac{p_{0 \lambda}^{\prime}}{\phi_{0}}\right)\left[\frac{3\left(2 \gamma_{1}-1\right)}{3 \gamma_{1}-1}+\frac{3\left(\gamma_{1}-1\right)^{2} \delta \tau(f)}{3 \gamma_{1}-1}-\frac{p_{0 \alpha}^{\prime}}{\phi_{0}}-\frac{1}{2} \frac{p_{0 \lambda}^{\prime}}{\phi_{0}}\right] .
$$

Using the Parsons-Lee approach in the framework of Onsager investigation for sufficiently long spherocylinders we determined that $\delta=3 / 8[16]$. As a result, we can present the constant $C$ in the form

$$
C^{\mathrm{CS}-\mathrm{PL}}=\frac{\eta_{1} / \phi_{0}}{1-\eta_{1} / \phi_{0}}\left[\frac{3\left(\gamma_{1}-1\right)^{2}}{3 \gamma_{1}-1}\left(1-\frac{p_{0 \lambda}^{\prime}}{2 \phi_{0}}\right)+\frac{\eta_{1} / \phi_{0}}{\left(1-\eta_{1} / \phi_{0}\right)} \delta \frac{\left(\gamma_{1}-1\right)^{2}}{3 \gamma_{1}-1}\left(\frac{6 \gamma_{1}}{3 \gamma_{1}-1}-\frac{p_{0 \lambda}^{\prime}}{\phi_{0}}\right)\right] .
$$




\section{Generalization of the Parsons-Lee theory for the hard spherocylinder fluid in disordered porous media}

In this section we generalize the PL theory for the case of hard spherocylinder fluid in disordered porous media. In [16] in the framework of the functional scaling concept, a direct generalization of the CS equation for the free energy of hard sphere fluid for a nematic fluid was constructed. Following [16], in accordance with 2.25 and (3.7), a generalized expression for the hard spherocylinder fluid in disordered porous media can be written as

$$
\begin{aligned}
& \left(\frac{\beta F}{N_{1}}\right)^{\mathrm{PL}}=\ln \left(\frac{\eta_{1}}{\phi}\right)-1+\sigma(f)+\left\{\left(1-\frac{\phi_{0}}{\phi}\right)\left[1+\frac{\phi_{0}}{\eta_{1}} \ln \left(1-\phi_{0} / \eta_{1}\right)\right]\right. \\
& \left.+\left(1+\frac{A}{2}\right) \frac{\eta_{1} / \phi_{0}}{1-\eta_{1} / \phi_{0}}+\left(\frac{B}{3}-\frac{1}{2}\right)\left(\frac{\eta_{1} / \phi_{0}}{1-\eta_{1} / \phi_{0}}\right)^{2}\right\}\left[1+\frac{3}{4} \frac{\left(\gamma_{1}-1\right)^{2}}{3 \gamma_{1}-1} \tau(f)\right],
\end{aligned}
$$

where

$$
\begin{gathered}
A=6+4 \frac{p_{0 \lambda}^{\prime}}{\phi_{0}}+\left(\frac{p_{0 \lambda}^{\prime}}{\phi_{0}}\right)^{2}-\frac{1}{2} \frac{p_{0 \lambda}^{\prime \prime}}{\phi_{0}}, \\
B=\frac{1}{2}\left(3-\frac{p_{0 \lambda}^{\prime}}{\phi_{0}}\right)^{2} .
\end{gathered}
$$

For the pressure and the chemical potential we will respectively have

$$
\begin{aligned}
& \left(\frac{\beta P}{\rho_{1}}\right)^{\mathrm{PL}}=1+\left\{\frac{\phi_{0}}{\phi} \frac{\eta_{1} / \phi_{0}}{1-\eta_{1} / \phi_{0}}+\left(\frac{\phi_{0}}{\phi}-1\right)\left[1+\frac{\phi_{0}}{\eta_{1}} \ln \left(1-\eta_{1} / \phi_{0}\right)\right]+\frac{A}{2} \frac{\eta_{1} / \phi_{0}}{\left(1-\eta_{1} / \phi_{0}\right)^{2}}\right. \\
& \left.+\frac{2 B}{3} \frac{\left(\eta_{1} / \phi_{0}\right)^{2}}{\left(1-\eta_{1} / \phi_{0}\right)^{3}}-\frac{\left(\eta_{1} / \phi_{0}\right)^{3}}{\left(1-\eta_{1} / \phi_{0}\right)^{3}}\right\}\left[1+\frac{3}{4} \frac{\left(\gamma_{1}-1\right)^{2}}{3 \gamma_{1}-1} \tau(f)\right], \\
& \left(\beta \mu_{1}\right)^{\mathrm{PL}}=\ln \left(\frac{\eta_{1}}{\phi}\right)+\sigma(f)+\left\{\left(1+\frac{\phi_{0}}{\phi}+A\right) \frac{\eta_{1} / \phi_{0}}{1-\eta_{1} / \phi_{0}}+\left[\frac{1}{2}(A-1)+B\right] \frac{\left(\eta_{1} / \phi_{0}\right)^{2}}{\left(1-\eta_{1} / \phi_{0}\right)^{2}}\right. \\
& \left.+\left(\frac{2 B}{3}-1\right) \frac{\left(\eta_{1} / \phi_{0}\right)^{3}}{\left(1-\eta_{1} / \phi_{0}\right)^{3}}\right\}\left[1+\frac{3}{4} \frac{\left(\gamma_{1}-1\right)^{2}}{3 \gamma_{1}-1} \tau(f)\right] .
\end{aligned}
$$

After minimization of the free energy, in the considered approach we obtain an integral equation for the singlet orientational distribution function in the form $(2.25)$, in which, however, the constant $C$ has the following form

$$
\begin{aligned}
C^{\mathrm{PL}} & =\frac{6}{\pi} \frac{\left(\gamma_{1}-1\right)^{2}}{3 \gamma_{1}-1}\left\{\left(1-\frac{\phi_{0}}{\phi}\right)\left[1+\frac{\phi_{0}}{\eta_{1}} \ln \left(1-\eta_{1} / \phi_{0}\right)\right]\left(1+\frac{A}{2}\right) \frac{\eta_{1} / \phi_{0}}{1-\eta_{1} / \phi_{0}}\right. \\
& \left.+\left(\frac{B}{3}-\frac{1}{2}\right)\left(\frac{\eta_{1} / \phi_{0}}{1-\eta_{1} / \phi_{0}}\right)^{2}\right\} .
\end{aligned}
$$

\section{Results and discussions}

We will illustrate the developed approaches for the hard spherocylinder fluid in a hard sphere matrix. First, we specify the geometrical and the probe particle porosities [26]. The geometrical porosity $\phi_{0}$ in this case has the form

$$
\phi_{0}=1-\eta_{0},
$$


where $\eta_{0}=\rho_{0} V_{0}, \rho_{0}=\frac{N_{0}}{V}, N_{0}$ is the number of matrix particles, $V_{0}=\frac{1}{6} \pi D_{0}^{3}$ is the volume of a matrix particle, $V$ is the total volume of the system, $D_{0}$ is the diameter of matrix hard spheres.

Using the SPT, the following expression for the probe particle porosity is derived [26]

$$
\begin{aligned}
\phi & =\left(1-\eta_{0}\right) \exp \left\{-\frac{\eta_{0}}{1-\eta_{0}} \frac{D_{1}}{D_{0}}\left[\frac{3}{2}\left(\gamma_{1}+1\right)+3 \gamma_{1} \frac{D_{1}}{D_{0}}\right]-\frac{\eta_{0}^{2}}{\left(1-\eta_{0}\right)^{2}} \frac{9}{2} \gamma_{1} \frac{D_{1}^{2}}{D_{0}^{2}}\right. \\
& \left.-\frac{\eta_{0}^{3}}{\left(1-\eta_{0}\right)^{3}}\left(3 \gamma_{1}-1\right) \frac{1}{2} \frac{D_{1}^{3}}{D_{0}^{3}}\left(1+\eta_{0}+\eta_{0}^{2}\right)\right\} .
\end{aligned}
$$

The probability to find a small scaled spherocylinder in an empty matrix is

$$
p_{0}\left(\alpha_{\mathrm{s}}, \lambda_{\mathrm{s}}\right)=1-\eta_{0} \frac{1}{V_{0}} \frac{\pi}{2}\left[\frac{1}{3}\left(D_{0}+\lambda_{\mathrm{s}} D_{1}\right)^{3}+\frac{1}{2} \alpha_{\mathrm{s}} L_{1}\left(D_{0}+\lambda_{\mathrm{s}} D_{1}\right)^{2}\right] .
$$

Hereupon we can find the derivatives needed for the description of thermodynamic properties of a confined fluid:

$$
p_{0 \lambda}^{\prime}=-3 \frac{D_{1}}{D_{0}} \eta_{0}, \quad p_{0 \alpha}^{\prime}=-\frac{3}{2} \eta_{0} \frac{L_{1}}{D_{0}}, \quad p_{0 \alpha \lambda}^{\prime \prime}=-3 \eta_{0} \frac{L_{1}}{D_{0}} \frac{D_{1}}{D_{0}}, \quad p_{0 \lambda \lambda}^{\prime \prime}=-6 \eta_{0} \frac{D_{1}^{2}}{D_{0}^{2}} .
$$

Now we apply the theory presented in the previous section for investigation of the isotropic-nematic phase transition in a hard spherocylinder fluid confined in a matrix formed by a disordered hard sphere. We start this study from the bifurcation analysis of the integral equation 2.25) for the singlet distribution function $f(\Omega)$. This equation has the same form as the corresponding equation obtained by Onsager [2] for the hard spherocylinder fluid in the limit of $L_{1} \rightarrow \infty, D_{1} \rightarrow 0$ while the dimensional density of fluid $c_{1}=\frac{1}{4} \pi \rho_{1} L_{1}^{2} D_{1}$ is fixed. In the Onsager limit

$$
C \rightarrow c_{1}=\frac{1}{4} \pi \rho_{1} L_{1}^{2} D_{1}
$$

From the bifurcation analysis of the integral equation 2.25 it was found that this equation has two characteristic points $C_{\mathrm{i}}$ and $C_{\mathrm{n}}$ [28], which define the range of stability of a considered system. The first point $C_{\mathrm{i}}$ corresponds to the highest value of a possible density of a stable isotropic state and the second point $C_{\mathrm{n}}$ corresponds to the lowest value of a possible density of a stable nematic state. For the Onsager model from the solution of the coexistence equations, the values of the density of coexisting isotropic and nematic phases were obtained [29,-31]

$$
c_{\mathrm{i}}=3.289, \quad c_{\mathrm{n}}=4.192 .
$$

For the finite values of $L_{1}$ and $D_{1}$ we can put

$$
C_{\mathrm{i}}=3.289, \quad C_{\mathrm{n}}=4.192 .
$$

For the constant $C$ in this paper we have three different approximations. In the SPT2b1 $C$ is given by the expression (2.26), for CS-PL approximation $C$ is given by the expression (3.9) and for PL approximation $C$ is given by the expression (4.6). The values $(5.7)$ for $C$ define the isotropic-nematic phase diagram for a hard spherocylinder fluid in disordered porous media depending on the ratio $L_{1} / D_{1}=\gamma_{1}-1$ and the parameter of the matrix, namely $\eta_{0}=1-\phi_{0}$ and the ratio $D_{1} / D_{0}$. To be more specific, we will fix the last ratio by putting $D_{0}=L_{1}$. As a result, $\frac{D_{1}}{D_{0}}=1 /\left(\gamma_{1}-1\right)$.

At the beginning we will demonstrate how the developed approaches describe the isotropic-nematic coexistence curves for a hard spherocylinder fluid in the bulk case. In figure 1 we present the dependence of the density $\eta_{1}$ on the parameter $\gamma_{1}$ along the isotropic-nematic coexistence curves obtained from the bifurcation analysis in SPT2b1, CS-PL and PL approximations. For comparison the computer simulation results taken from [32, 33] are presented as well. As we can see all three approximations for large enough values of $\gamma_{1}$ give the same results in good agreement with the simulation data. However, starting 


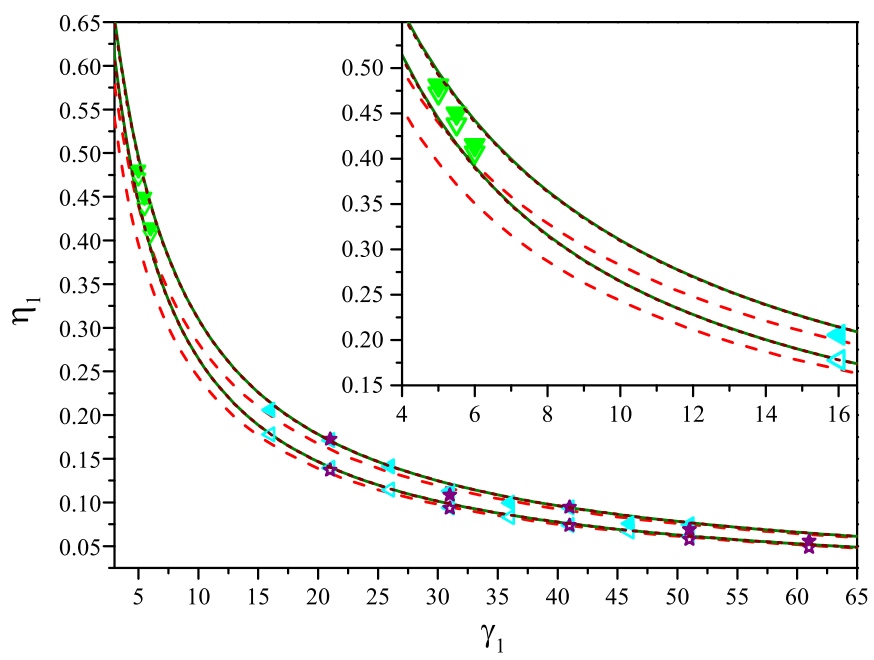

Figure 1. (Colour online) Isotropic-nematic coexistence diagram in the bulk case for a hard spherocylinder fluid in the plane of the packing fraction of the fluid $\eta_{1}$ versus parameter $\gamma_{1}$. The results presented are obtained from the bifurcation analysis of the integral equation 2.25 in different approximations for the constant $C$ : red dashed line denotes SPT2b1, green solid line denotes PL, brown dotted line denotes CS-PL, down pointed green triangles $\nabla$ are the results of the simulations [32], left pointed triangles $\triangleleft$ are GDI simulation results [33] and star points $\star$ are the GEMC simulation results taken from [33].

from $\gamma_{1}$ near $\gamma_{1} \approx 30$ there is a deviation of the SPT2b1 approximation from general tendency and the computer simulation data. This deviation increases with a decreasing parameter $\gamma_{1}$ and at $\gamma_{1}$ smaller than 20 it leads to incorrect results. Two other approximations, namely the CS-PL and PL ones, reproduce more correctly the general tendency of the dependence of coexistence curves on $\gamma_{1}$. We do not observe differences between the CS-PL and PL approximations. However, at small $\gamma_{1}$, the results predicted from the bifurcation analysis slightly overestimate the jump of the density at the phase transition.

We should note that the isotropic-nematic coexistence lines can also be found from the condition of thermodynamic equilibrium, according to which the isotropic and nematic phases have the same pressure and the same chemical potential:

$$
P\left(\eta_{\mathrm{i}}\right)=P\left(\eta_{\mathrm{n}}\right), \quad \mu\left(\eta_{\mathrm{i}}\right)=\mu_{1}\left(\eta_{\mathrm{n}}\right) .
$$

The coexistence curves obtained from the condition (5.8) for a hard spherocylinder fluid in the bulk case are presented in figure 2. As it was shown in [28], in the Onsager limit, the results obtained from the bifurcation analysis and from the condition of thermodynamic equilibrium coincide exactly. Similar to the bifurcation analysis in the thermodynamic way for large enough values of $\gamma_{1}$ all three approximations give the same results, but with a decreasing $\gamma_{1}$ we observed a deviation in SPT2b1 approximation and computer simulation data which leads to incorrect results at small $\gamma_{1}$. Again we do not observe the difference between the CS-PL and PL results. However, at small $\gamma_{1}$, contrary to the bifurcation analysis, the thermodynamic consideration slightly underestimates the value of the density jump between the isotropic and nematic phases. Nevertheless, comparing figure 1 and figure 2 we can see that thermodynamic consideration leads to a better agreement with the computer simulation data.

As we have already noted in the Onsager limit all three approximations in the thermodynamic approach and in the bifurcation analysis reproduce correctly the exact result (5.6). In the presence of a porous medium for the Onsager model we obtain

$$
c_{\mathrm{i}} / \phi_{0}=3.289, \quad c_{\mathrm{n}} / \phi_{0}=4.192 .
$$

It means that for the isotropic-nematic phase transition, the presence of a porous medium shifts the phase diagram to lower densities of a fluid. This effect of the porous medium is illustrated in figure 3 where the dependence of the density of fluid $\eta_{1}$ on parameter $\gamma_{1}$ along the isotropic-nematic coexistence curves calculated from thermodynamic equilibrium (5.8) for the hard spherocylinder fluid in a porous 


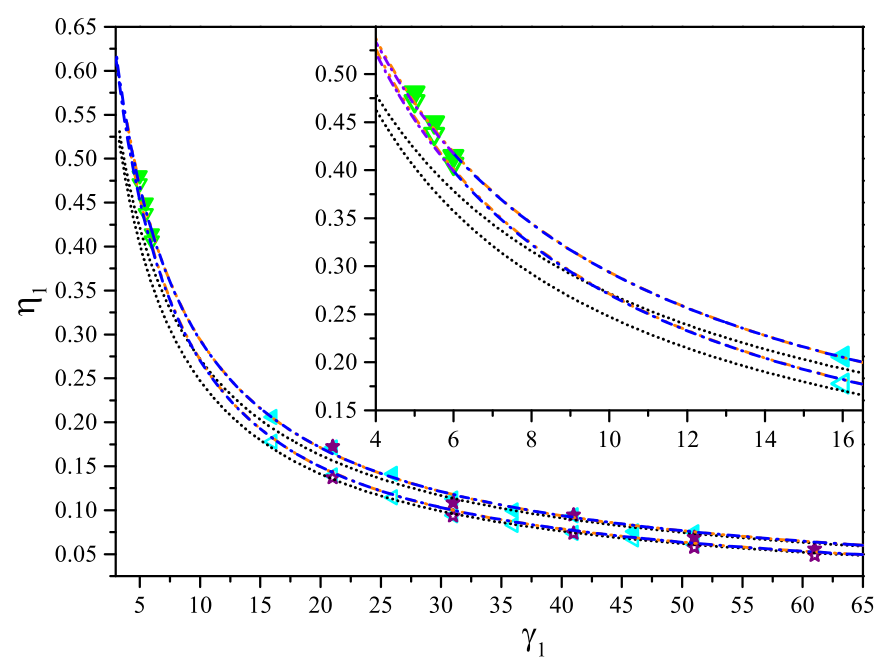

Figure 2. (Colour online) Isotropic-nematic coexistence diagram in the bulk case for a hard spherocylinder fluid in the plane of the packing fraction of the fluid $\eta_{1}$ versus parameter $\gamma_{1}$. The results presented are obtained from the thermodynamics analysis in different approximations: black dotted line denotes SPT2b1, orange dash-dot-dot line denotes PL, blue dash-dot-dash line denotes CS-PL, down pointed green triangles $\nabla$ are the results of simulations taken from [32], left pointed triangles $\triangleleft$ are GDI simulations results from [33] and star points $\star$ are the GEMC simulations results taken from [33].

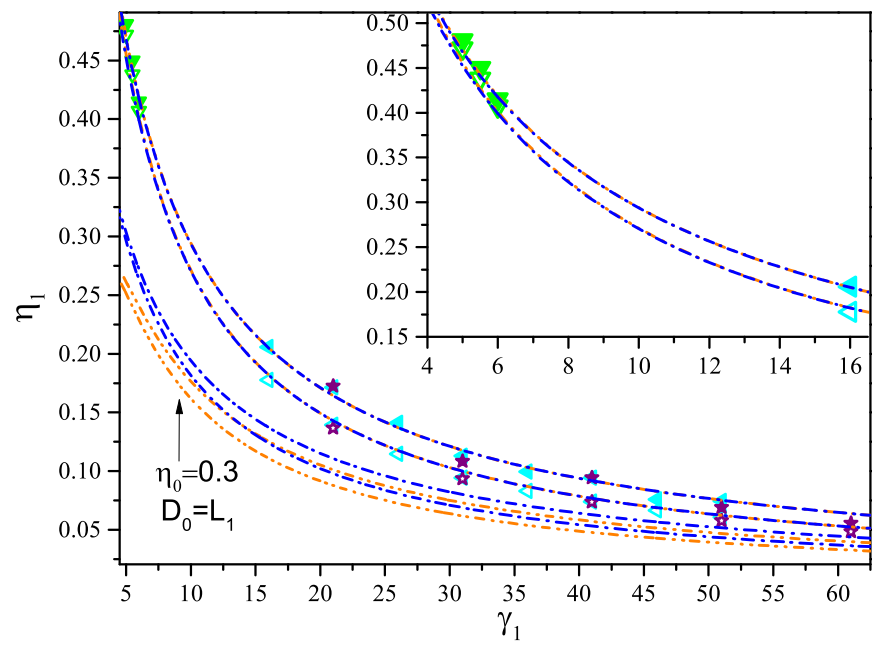

Figure 3. (Colour online) The influence of the porous medium on the isotropic-nematic coexistence diagram for a hard spherocylinder fluid in the plane of the packing fraction of the fluid $\eta_{1}$ versus parameter $\gamma_{1}$. The results are calculated from the thermodynamics analysis. The results for a fluid in the disordered porous medium with the porosity $\phi_{0}=0.7\left(\eta_{0}=0.3\right)$ are presented by dotted lines. For comparison purposes the results for the bulk case are also presented. The notations are the same as in figure 2

medium with the porosity $\phi_{0}=0.7\left(\eta_{0}=0.3\right)$ is presented. For comparison, the isotropic-nematic diagram for the bulk case is also presented.

The influence of porosity $\phi_{0}$ on the coexistence lines of the isotropic-nematic phase transition calculated from the conditions $(5.8)$ for a hard spherocylinder fluid in coordinates fluid density $\eta_{1}$ versus packing fraction $\eta_{0}$ of matrix particles (the porosity $\phi_{0}=1-\eta_{0}$ ) is illustrated also in figure 4 and figure 5 for the cases $L_{1} / D_{1}=20$ and $L_{1} / D_{1}=5$, correspondingly. All the curves are obtained from the condition of thermodynamic equilibrium (5.8) in CS-PL and PL approximations, correspondingly. As we can see there are small insignificant differences between the predictions from both approaches. For 


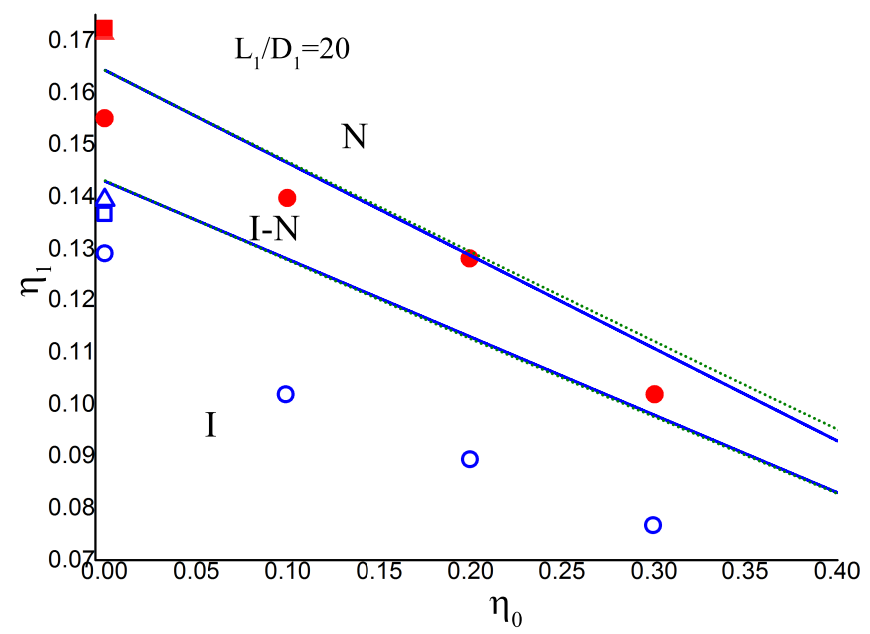

Figure 4. (Colour online) Coexistence lines of isotropic-nematic phases of a hard spherocylinder fluid in a hard sphere matrix for $L_{1} / D_{1}=20$ and $D_{0} / L_{1}=1$. Dependencies of the spherocylinder fluid packing fraction $\eta_{1}$ on the matrix packing fraction $\eta_{0}$ are presented. The results are obtained from the thermodynamics analysis with green dotted lines corresponding to the PL approximation and the blue solid lines corresponding to the CS approximation. The GEMC results taken from [34] are shown as circles and those taken from [33] are shown as squares and triangles (GDI).

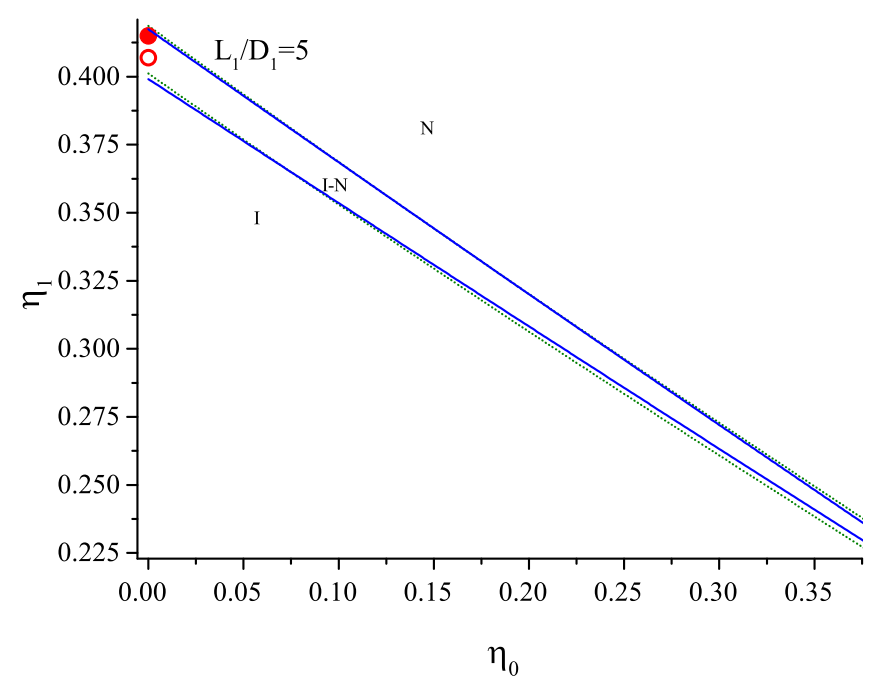

Figure 5. (Colour online) Coexistence lines of isotropic-nematic phases of a hard spherocylinder fluid in a hard sphere matrix for $L_{1} / D_{1}=5$ and $D_{0} / L_{1}=1$. Dependencies of the spherocylinder fluid packing fraction $\eta_{1}$ on the matrix packing fraction $\eta_{0}$ are presented. The results are obtained from the thermodynamics analysis with green dotted lines corresponding to the PL approximation and the blue solid lines corresponding to the CS approximation. The GEMC results taken from [32] are shown as red circles.

comparison, in figure 4 the results of the computer simulations of Schmidt and Dijkstra [34] obtained by the method of Gibbs ensemble Monte Carlo (GEMC) are also presented. For the bulk case $\left(\eta_{0}=0\right)$, the results of Bolhuis and Frenkel [33] obtained by GEMC method and GEMC combined with modified Gibbs-Duhem integration (GDI) method are shown as well. As we can see there are some differences between the computer data from [34] and [33]. Our theoretical prediction is in better agreement with the data from [33] and correctly reproduces the dependences of $\eta_{1}$ on $\eta_{0}$ along the coexistence curves. The computer simulation results from [32] are also presented in figure 5. We see a good correlation between computer simulation data and theoretical prediction. 


\section{Conclusions}

In this paper the scaled particle theory (SPT) is extended for the description of a hard spherocylinder fluid in a disordered porous medium. We started from the SPT2b1 approach previously developed by us [21, 22] for a hard sphere fluid in a disordered porous medium and generalized in [26] for a hard spherocylinder fluid. The theory in this paper is applied for the study of the influence of disordered porous media on the isotropic-nematic transition in a hard spherocylinder fluid. It is shown that the accuracy of the SPT2b1 decreases with decreasing lengths of spherocylinders. Two different approaches are developed in order to improve the SPT2b1 theory. In one of them, the so-called SPT2b1-CSPL approach, two corrections are involved. The first one is the Carnahan-Starling correction which improves SPT description of thermodynamical properties at higher densities of the fluid. The second one corrects the description of orientational ordering in a hard spherocylinder fluid at higher densities. The constant of this correction is obtained from the comparison of the integral equation for the singlet distribution function of a hard spherocylinder fluid in the SPT2b1 and Parsons-Lee (PL) approaches. In the second approach, the so-called SPT2b1-PL approach, the PL theory [16] is generalized for a hard spherocylinder fluid in a disordered porous medium. To this end, according to the original PL theory [16], thermodynamic properties of a hard spherocylinder fluid in a disordered porous medium are mapped with the thermodynamic properties of a hard sphere fluid in a disordered porous medium in the SPT2b1 approximation [21, 22] with the CS correction considered in this paper.

The phase diagram of a hard spherocylinder fluid in a disordered porous medium is calculated in two different ways. One of them is connected with the bifurcation analysis of the nonlinear integral equation for the singlet distribution function obtained from minimization of the free energy of the considered system. The second way is based on the condition of thermodynamic equilibrium. The obtained results are compared with the existing computer simulation data [32-34]. It is shown that in both approaches the original SPT2b1 approximation is not very accurate with the decreasing length of spherocylinders. The SPT2b1-CS-PL and SPT2b1-PL approximations in the bifurcation analysis and in thermodynamic way more or less correctly reproduce the coexistence curves with decreasing lengths of spherocylinders. We do not find a significant difference between the SPT2b1-CS-PL and SPT2b1-PL approximations. However, the bifurcation analysis slightly overestimates the change of density at the phase transition. In thermodynamic way, we also did not find the best agreement between theoretical prediction and computer simulation data for small enough lengths of spherocylinders. In any case, the thermodynamic way is more preferable for the description of a phase transition. From bifurcation analysis and from thermodynamic way we show that the porous medium shifts the phase diagram to lower densities of a fluid. Comparison with computer simulation results is discussed.

The model considered in this paper can be used as the reference system for generalization of the Van der Waals theory for anisotropic fluids in disordered porous media [26, 27, 35] and for taking different types of associations [36] into account.

\section{References}

1. Vroege G.J., Lekkerkerker H.N.W., Rep. Prog. Phys., 1992, 55, 1241, doi 10.1088/0034-4885/55/8/003

2. Onsager L., Ann. N.Y. Acad. Sci., 1949, 51, 627, doi 10.1111/j.1749-6632.1949.tb27296.x

3. Reiss H., Frisch H.L., Lebowitz J.L., J. Chem. Phys., 1959, 31, 369, doi:10.1063/1.1730361

4. Reiss H., Frisch H.L., Helfand E., Lebowitz J.L., J. Chem. Phys., 1960, 32, 119, doi:10.1063/1.1700883

5. Cotter M.A., Martire D.E., J. Chem. Phys., 1970, 52, 1909, doi $10.1063 / 1.1673232$

6. Cotter M.A., Phys. Rev. A, 1974, 10, 625, doi $10.1103 /$ PhysRevA.10.625

7. Cotter M.A., Wacker D.C., Phys. Rev. A, 1978, 18, 2669, doi:10.1103/PhysRevA.18.2669

8. Thiele E., J. Chem. Phys., 1963, 39, 474, doi $10.1063 / 1.1734272$

9. Wertheim M.S., Phys. Rev. Lett., 1963, 10, 321, doi $10.1103 /$ PhysRevLett.10.321.

10. Yukhnovski I.R., Holovko M.F., Statistical Theory of Classical Equilibrium Systems, Naukova Dumka, Kyiv, 1980, (in Russian).

11. Carnahan N.F., Starling K.E., J. Chem. Phys., 1969, 51, 635, doi $10.1063 / 1.1672048$

12. Holovko M.F., Hvozd M.V., Condens. Matter Phys., 2017, 20, 43501, doi 10.5488/CMP.20.43501

13. Lago S., Cuetos A., Martínez-Haya B., Rull L.F., J. Mol. Recognit., 2004, 17, 417, doi 10.1002/jmr.704 
14. Wu L., Malijevský A., Jackson G., Müller E.A., Avendaño C., J. Chem. Phys., 2015, 143, 044906, doi $10.1063 / 1.4923291$

15. Parsons J.D., Phys. Rev. A, 1979, 19, 1225, doi $10.1103 /$ PhysRevA.19.1225

16. Lee S.D., J. Chem. Phys., 1987, 87, 4972, doi $10.1063 / 1.452811$

17. Lee S.D., J. Chem. Phys., 1988, 89, 7036, doi:10.1063/1.455332

18. Holovko M., Dong W., J. Phys. Chem. B, 2009, 113, 6360, doi $10.1021 /$ jp809706n

19. Chen W., Dong W., Holovko M., Chen X.S., J. Phys. Chem. B, 2010, 114, 1225, doi $10.1021 /$ jp9106603

20. Patsahan T., Holovko M., Dong W., J. Chem. Phys., 2011, 134, 074503, doi 10.1063/1.3532546

21. Holovko M., Patsahan T., Dong W., Condens. Matter. Phys., 2012, 15, 23607, doi $10.5488 / C M P .15 .23607$

22. Holovko M., Patsahan T., Dong W., Pure Appl. Chem., 2013, 85, 115, doi 10.1351/PAC-CON-12-05-06

23. Chen W., Zhao S.L., Holovko M.F., Chen X.S., Dong W., J. Phys. Chem. B, 2016, 120, 5491, doi $10.1021 /$ acs.jpcb.6b02957.

24. Holovko M.F., Patsahan T., Dong W., Condens. Matter. Phys., 2017, 20, 33602, doi 10.5488/CMP.20.33602

25. Holovko M., Shmotolokha V., Patsahan T., J. Mol. Liq., 2014, 189, 30, doi 10.1016/j.molliq.2013.05.030

26. Holovko M., Shmotolokha V., Patsahan T., In: Physics of Liquid Matter: Modern Problems, Springer Proceedings in Physics, Vol. 171, Bulavin L., Lebovka N. (Eds.), Springer, Heidelberg, 2015, 3-30.

27. Holovko M., Shmotolokha V., Ukr. J. Phys., 2015, 60, 770, doi $10.15407 /$ ujpe60.08.0770

28. Kayser R.F. (Jr.), Raveché H.J., Phys. Rev. A, 1978, 17, 2067, doi 10.1103/PhysRevA.17.2067

29. Herzfeld J., Berger A.E., Wingate J.W., Macromolecules, 1984, 17, 1718, doi $10.1021 / \mathrm{ma00139a014}$

30. Lekkerkerker H.N.W., Coulon Ph., Van Der Haegen R., Deblieck R., J. Chem. Phys., 1984, 80, 3427, doi $10.1063 / 1.447098$

31. Chen Z.Y., Macromolecules, 1993, 26, 3419, doi:10.1021/ma00065a027

32. McGrother S.C., Williamson D.C., Jackson G., J. Chem. Phys., 1996, 104, 6755, doi 10.1063/1.471343

33. Bolhuis P., Frenkel D., J. Chem. Phys., 1997, 106, 666, doi:10.1063/1.473404

34. Schmidt M., Dijkstra M., J. Chem. Phys., 2004, 121, 12067, doi $10.1063 / 1.1815294$

35. Holovko M., Shmotolokha V., Patsahan T., Condens. Matter Phys., 2015, 18, 13607, doi:10.5488/CMP.18.13607

36. Kalyuzhnyi Y.V., Holovko M., Patsahan T., Cummings P.T., J. Phys. Chem. Lett., 2014, 5, 4260, doi $10.1021 / \mathrm{jz} 502135 \mathrm{f}$

\title{
Теорія масштабної частинки для системи сфероциліндричного плину в невпорядкованому пористому середовищі: поправки Карнагана-Старлінга і Парсонса-Лі
}

\author{
М.Ф. Головко, В.І. Шмотолоха \\ Інститут фізики конденсованих систем НАН України, вул. І. Свєнціцького, 1, 79011 Львів, Україна
}

Теорія масштабної частинки (ТМЧ) застосовується для вивчення впливу пористого середовища на ізотропно-нематичний перехід у плині твердих сфероциліндрів. Розроблено два нові підходи для покращення опису сфероциліндрів невеликої довжини. В одному з них, так званому підході ТМЧ-КС-ПЛ, вводиться поправка Карнагана-Старлінга (КС) для покращення опису термодинамічних властивостей плину, тоді як поправка Парсонса-Лі (ПЛ) покращує опис орієнтаційного впорядкування. Другий підхід, так званий підхід ТМЧ-ПЛ, пов'язаний з узагальненням теорії Парсонса-Лі для анізотропних рідин у невпорядкованих пористих середовищах. Фазова діаграма отримана з біфуркаційного аналізу нелінійного інтегрального рівняння для одночастинкової функції розподілу та умови термодинамічної рівноваги. Отримані дані порівнюються з даними комп'ютерних симуляцій. Обидва шляхи і обидва підходи істотно покращують опис системи сфероциліндричного плину у випадку малих довжин сфероциліндра. Ми не знайшли істотної різниці в результатах в обох розроблених підходах. Ми виявили, що біфуркаційний аналіз трохи переоцінює, а термодинамічний аналіз недооцінює передбачення, отримані з комп'ютерних симуляцій. Пористе середовище зсуває фазову діаграму в бік менших густин плину і не змінює тип переходу.

Ключові слова: твердий сфероциліндричний плин, пористий матеріал, теорія масштабної частинки, ізотропно-нематичний перехід, теорія Парсонса-Лі, поправка Карнагана-Старлінга 


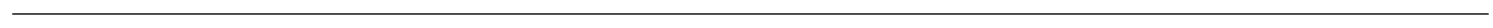

